\title{
El derecho a la educación en la medición de pobreza: un análisis complejo*
}

\author{
Manuel Lara Caballero ${ }^{1}$ \\ (D) http://orcid.org/0000-0002-5312-3085 \\ Universidad Autónoma Metropolitana, Unidad Lerma, México
}

DOI: http://dx.doi.org/10.17081/eduhum.19.33.2651

Recibido: 8 de enero de 2017

Aceptado: 5 de mayo de 2017

\section{The right to education when measurig powerty: a complete asalysis}

\author{
Palabras clave: \\ Derecho a la educación, \\ Medición de la pobreza, \\ Complejidad.
}

Key words:

Right to education, Poverty measurements, Complexity.

\begin{abstract}
Resumen
El objetivo de este artículo es realizar observaciones de primer y segundo orden, desde las ciencias de la complejidad, al indicador que el Consejo Nacional de Evaluación de la Política de Desarrollo Social (CONEVAL) utiliza para conceptualizar y medir el derecho a la educación en la metodología multidimensional de la pobreza, con la finalidad de realizar una propuesta preliminar que contribuya a resolver la forma de aproximarse a su medición. La metodología utilizada fue una adaptación de Amozurrutia (2012), en la que se realiza una aproximación epistemológica con base en los niveles de observación de primero y segundo orden de Luhmann (2007). Los resultados indican que el carácter de indivisibilidad e interdependencia del derecho a la educación conforman un factor que posibilita la complejidad, por lo cual se tiene que tomar en cuenta su interrelación con otros derechos sociales establecidos en la Ley General de Desarrollo Social.
\end{abstract}

\begin{abstract}
The aim of this article is to make first and second order observations, from the sciences of complexity, to the indicator that the National Council for the Evaluation of Social Development Policy (CONEVAL) uses for conceptualizing and measuring the right to education in the multidimensional measurement of poverty, in order to make a preliminary proposal that may contribute to the discussion of how to approach a way for its measurement. The methodology utilized was an adaptation of a proposal from Amozurrutia (2012) using an epistemological approach based on the observations of first and second level of Luhmann (2007). The results of the analysis indicate that the indivisibility and interdependence features of the rights to education are factors that enable complexity, but it is important to take into account its inter relationship with other social rights established in the General Law of Social Development.
\end{abstract}

\section{$(1) \Theta \Theta$}

Referencia de este artículo (APA): Lara, M. (2017). El derecho a la educación en la medición de pobreza: un análisis complejo. En Revista Educación y Humanismo, 19(33), 386-397. http://dx.doi.org/10.17081/eduhum.19.33.2651

* El artículo hace parte de la línea de investigación Medición Económica financiado por la Universidad Autónoma Metropolitana, Unidad Lerma, México.

1. Profesor-investigador del Departamento de Procesos Sociales. Universidad Autónoma Metropolitana, Unidad Lerma. m.lara@correo.ler.uam.mx 


\section{Introducción}

La educación juega un papel crucial en el desarrollo económico, político y social, por lo que la mayor parte de los países han intentado, a través de diversos marcos normativos, nacionales e internacionales, establecerlo como un derecho fundamental presente en la política social (Ibagón, 2015).

En el contexto de México, se publicó la Ley General de Desarrollo Social (LGDS) en el año 2004, que permitió un acercamiento al problema de la pobreza con la finalidad de garantizar a toda la población el ejercicio pleno de los derechos sociales establecidos en la Constitución Política de los Estados Unidos Mexicanos (CPEUM).

La LGDS establece que la educación es un derecho central para el desarrollo social y, por lo tanto, es necesario contar con mecanismos, instituciones y reglas para hacer seguimiento a las diversas acciones del gobierno y de la sociedad civil al respecto. Por tal motivo, fue importante la construcción de la medición multidimensional de la pobreza, una metodología oficial elaborada por el Consejo Nacional para la Evaluación de la Política de Desarrollo Social (CONEVAL), que permite no solo el reconocimiento de los derechos sociales, sino también identificar y conocer la situación de pobreza en la que viven millones de mexicanos.

La estrategia nacional contra la pobreza ha enfrentado no solo la dificultad de su aproximación técnico-política desde la administración pública, sino de su comprensión como fenómeno complejo porque el CONEVAL utilizó un enfoque que puede parecer reduccionista $\mathrm{y}$ facilitar la intervención gubernamental -en tanto acción pública concreta y observable-, pero está lejos de demostrar mejoras en los niveles de bienestar de la población más vulnerable y, sobre todo, de garantizar el goce pleno de derechos sociales. En el proceso de simplificación de los enfoques de intervención se pierde de vista, en efecto, la complejidad constitutiva de la pobreza, generando estrategias lineales que han demostrado su ineficiencia al tratar de contener este fenómeno.

En este marco, el objetivo de esta investigación consiste en realizar observaciones de primer y segundo orden, desde las ciencias de la complejidad, al indicador que el CONEVAL utiliza para conceptualizar y medir el derecho a la educación en la metodología multidimensional de la pobreza. Esto con la finalidad de contribuir a la discusión acerca de si el derecho a la educación tendría que estar interrelacionado con otros elementos (salud, vivienda, alimentación, etcétera), aspecto que se pierde de vista en la implementación de las estrategias gubernamentales para reducir los índices de pobreza.

El artículo dispone de cuatro apartados. En el primero, se describe el problema de investigación, es decir, el indicador del derecho a la educación en la medición multidimensional de la pobreza elaborado por el CONEVAL. En el segundo, se presenta una propuesta metodo- 
lógica con base en Amozurrutia (2012), la cual permitirá analizar el derecho social a la educación. En el tercer apartado, se abordan los resultados del análisis para contribuir a la discusión sobre cómo acercarse a la medición del derecho a la educación. En este se propone que para garantizar los derechos sociales, estos tienen que ser vistos de manera interrelacionada, entretejida, religada, es decir, como un complexus dinámico, trasladando la discusión a nivel de política pública como un Todo que no solo es mayor a sus partes, sino que también es menos que la suma de ellas (Morin, 2008). Por último, con la intención de poner en perspectiva el ejercicio realizado, se plantean discusiones y conclusiones.

\section{Problema de investigación}

Los derechos humanos son inherentes a todos, sin distinción de nacionalidad, lugar de residencia, sexo, color, religión, idioma o cualquier otra condición; son universales porque todos pueden participar de ellos, y progresivos, por estar en constante evolución y no permitir un retroceso. Además, se encuentran contemplados y garantizados en la ley a través de los tratados, el derecho internacional consuetudinario, los principios generales y otras fuentes del derecho internacional. La esencia de los derechos humanos se encuentra en los principios establecidos por el derecho (Oficina del Alto Comisionado para los Derechos Humanos, 2004):

- El principio de indivisibilidad. Reconoce que ningún derecho es intrínsecamente inferior a ningún otro, por tanto los derechos económicos, sociales y culturales deben ser respetados, protegidos y realizados en las mismas condiciones que los derechos civiles y políticos.

- El principio de interdependencia. Reconoce la dificultad de hacer efectivo cualquiera de los derechos de forma aislada respecto de los demás. Por ejemplo, es insuficiente hablar del derecho al trabajo sin que se haya hecho mínimamente efectivo el derecho a la educación básica.

Los principios de indivisibilidad y de interdependencia obligan a realizar esfuerzos para que los derechos humanos se hagan efectivos simultáneamente, estableciendo prioridades en los contextos de recursos escasos. Así, todos ellos forman una unitas multiplex en la que se relacionan unos con los otros de manera profunda, de tal suerte que constituyen un compendio imposible de fragmentar (Morin, 1999). De este modo, no es posible prescindir de uno solo de ellos sin afectar al resto. Por el contrario, cada derecho humano precisa de los demás para lograr su completa eficacia.

Entonces, ¿cómo abordar el análisis del derecho a la educación? Si bien la respuesta a esta pregunta no es fácil, nuestra perspectiva pretende analizar la relación construida analíticamente entre pobreza y educación a través de la metodología multidimensional para la medición de la pobreza, una aproximación de carácter oficial y técnica elaborada por el CONEVAL con 
base en los derechos sociales establecidos en la LGDS.

El punto de partida se encuentra en los indicadores que la LGDS, en los artículos $36 \mathrm{y}$ 37, establece al CONEVAL (2014) para medir la pobreza: I) el ingreso corriente per cápita; II) el rezago educativo promedio en el hogar; III) el acceso a los servicios de salud; IV) el acceso a la seguridad social; V) la calidad y espacios de la vivienda; VI) el acceso a los servicios básicos en la vivienda; VII) el acceso a la alimentación, y VIII) el grado de cohesión social.

Los elementos relevantes involucrados en la medición multidimensional de la pobreza son cuatro (CONEVAL, 2014): I) la definición del concepto de pobreza multidimensional; II) la construcción de la dimensión asociada al espacio de bienestar económico en la que se compara el ingreso corriente per cápita con una canasta alimentaria y no alimentaria; III) la identificación de las carencias de seis derechos sociales establecidos en la LGDS, que se miden mediante un índice de privación social, y IV) la combinación del ingreso y el índice de privación social para identificar los tipos de pobres.

El reto es analizar el enfoque del bienestar económico que representa el ingreso de los hogares como una aproximación a la capacidad que tienen para satisfacer sus necesidades alimentarias y no alimentarias, mientras se integra también al análisis el enfoque de los derechos asociado a la existencia de garantías fundamentales, inalienables, insustituibles e interdependientes. Con esta perspectiva, la pobreza puede ser una negación de los derechos humanos, dado que la pobreza como Todo se ve afectada por una de sus partes (impedimentos técnico-políticos en la implementación del derecho a la educación). Por esta razón, no se trata de romper el principio aristotélico de que el Todo es mayor a la suma de las partes, sino ir más allá: el Todo también es menor a la suma de las partes, puesto que un elemento constitutivo del Todo -en su interrelación con los demás- determina el resultado esperado (Morin, 2008).

Por otra parte, el enfoque de bienestar económico busca identificar las condiciones que limitan la libertad de las personas para realizarse plenamente; supone que cada persona, a partir de sus circunstancias y preferencias, desarrolla el conjunto de capacidades que definen el abanico de opciones de vida que puede elegir. Si estas opciones no le permiten tener condiciones de vida aceptables dentro de su sociedad, se considera que el individuo es pobre (CONEVAL, 2014).

El enfoque de los derechos sociales, además de otorgar un marco ético para la evaluación de las políticas de desarrollo social, proporciona un marco normativo con consecuencias prácticas en la definición de las responsabilidades sociales y el diseño de políticas públicas (Oficina del Alto Comisionado para los Derechos Humanos, 2004); también permite tomar en cuenta normas 
mínimas, principios y reconocimientos legales, no solo con respecto a los resultados, sino también durante el proceso mismo de implementación de estrategias de superación de la pobreza.

Debido a lo anterior, el último paso en la medición multidimensional de la pobreza consiste en el análisis simultáneo de ambos espacios para identificar a los individuos pobres. En el rectángulo de la Figura 1 se representa la población total. De este modo, en el eje vertical se tiene la primera dimensión que marca la LGDS, que es el ingreso neto corriente per cápita; mientras que en el eje horizontal se mide, de derecha a izquierda, el conjunto de las seis carencias sociales: educación, salud, vivienda, seguridad social, servicios básicos y alimentación; el número 0 indica la población que no tiene ninguna carencia, el número 6 significa que tiene todas las carencias de derechos sociales.

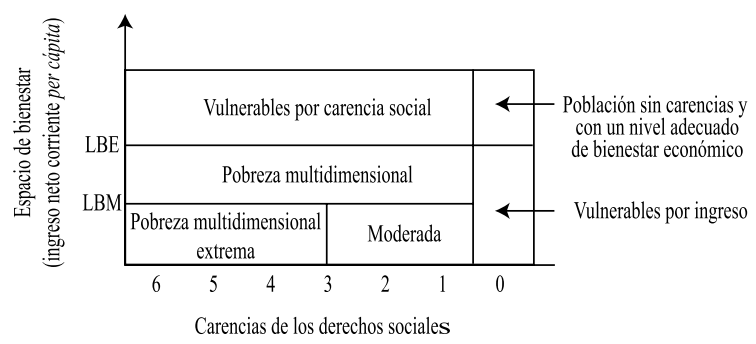

Figura 1. Población por tipo de pobreza

Nota: LBE significa Línea de Bienestar Económico y LBM representa la Línea de Bienestar Mínimo.

Fuente: elaboración propia con información del CONEVAL (2014)

Se identifican también los distintos tipos de pobres según la medición multidimensional de la pobreza (CONEVAL, 2014):

- Pobres multidimensionales: es la población con un ingreso por debajo de la línea de bienestar económico $(\$ 1.921,74$ para las zonas urbanas y $\$ 1.202,80$ para las zonas rurales) y que padece al menos una carencia social.

- Vulnerables por carencia social: se refiere a la población que presenta una o más carencias sociales, pero tiene un ingreso superior a la línea de bienestar económica.

- Vulnerables por ingreso: estos individuos no presentan carencias sociales, pero su ingreso es inferior o igual a la línea de bienestar mínimo.

- No pobre multidimensional y no vulnerable: población cuyo ingreso es superior a la línea de bienestar y no reporta ninguna carencia social.

Con la metodología multidimensional de la pobreza, el CONEVAL pretende dar un tratamiento complejo a la relación entre pobreza y educación, donde la educación parte de un todo más amplio y es vista como un elemento que se interrelaciona a su vez con las otras variables y derechos sociales con los que se puede medir la pobreza. Por ejemplo, la educación es una variable que explica, en parte, el nivel de ingresos de un hogar o individuo, donde a mayor educación, en promedio, se espera acceso a empleos mejor remunerados en el sector formal de la economía que por lo general pagan mejores salarios. Esto, a su vez, se relaciona con el tipo de vivienda que se adquiere, el acceso a la seguridad social, la alimentación, entre otros; la interdependencia de 
la educación con otras variables es lo que lleva a la complejidad.

En la medición multidimensional de la pobreza, el indicador que analiza el derecho a la educación fue definido como "rezago educativo" y para la construcción de su umbral se consultó al Instituto Nacional para la Evaluación de la Educación (INEE), organismo encargado de la evaluación del sector educativo en México. El INEE propuso al CONEVAL la Norma de Escolaridad Obligatoria del Estado Mexicano (NEOEM), conforme a la cual se considera como carencia por rezago educativo a la población que no cumpla con alguno de los siguientes criterios (CONEVAL, 2014):

- Tiene de tres a quince años, no cuenta con la educación básica obligatoria y no asiste a un centro de educación formal.

- Nació antes de 1982 y no cuenta con el nivel de educación obligatoria vigente en el momento en que debía de haberla cursado, es decir, la primaria completa.

- Nació a partir de 1982 y no cuenta con el nivel de educación obligatoria vigente, es decir, secundaria.

En resumen, la forma en que el CONEVAL mide si se cumple con el derecho a la educación, se limita solo al rezago educativo definido como la capacidad que tiene el Estado mexicano para lograr que la población tenga acceso o no a la educación básica obligatoria para toda su población.
Cuadro 1. Estimaciones del rezago educativo en México, 2012

\begin{tabular}{|l|c|l|c|}
\hline \multicolumn{1}{|c|}{$\begin{array}{c}\text { Entidades } \\
\text { federativas }\end{array}$} & $\begin{array}{c}\text { Porcentaje } \\
\mathbf{\%}\end{array}$ & $\begin{array}{c}\text { Entidades } \\
\text { federativas }\end{array}$ & $\begin{array}{c}\text { Porcentaje } \\
\mathbf{\%}\end{array}$ \\
\hline Aguascalientes & 15,3 & Morelos & 19,2 \\
\hline Baja California & 14,6 & Nayarit & 19,3 \\
\hline $\begin{array}{l}\text { Baja California } \\
\text { Sur }\end{array}$ & 15,7 & Nuevo León & 12,7 \\
\hline Campeche & 19,2 & Oaxaca & 27,7 \\
\hline Coahuila & 12,5 & Puebla & 24,1 \\
\hline Colima & 18,8 & Querétaro & 17,5 \\
\hline Chiapas & 33,5 & Quintana Roo & 17,6 \\
\hline Chihuahua & 16,1 & San Luis Potosí & 21,2 \\
\hline Distrito Federal & 9,2 & Sinaloa & 18,4 \\
\hline Durango & 16,1 & Sonora & 13,6 \\
\hline Guanajuato & 23,9 & Tabasco & 19,1 \\
\hline Guerrero & 26,8 & Tamaulipas & 15,5 \\
\hline Hidalgo & 20,6 & Tlaxcala & 15,8 \\
\hline Jalisco & 18,4 & Yucatán & 23,4 \\
\hline $\begin{array}{l}\text { Estado de } \\
\text { México }\end{array}$ & 15,4 & Veracruz & 25,8 \\
\hline Michoacán & 26,1 & Zacatecas & 21,1 \\
\hline Nivel nacional & & & 19,2 \\
\hline
\end{tabular}

Fuente: Elaboración propia con base en el CONEVAL, información del Módulo de Condiciones Socioeconómicas del Instituto de Estadística y Geografía (MCS de la ENIGH)

Los resultados del indicador de rezago educativo muestran que a nivel nacional, el $19,2 \%$ de la población tiene carencias en el derecho social a la educación. Es importante señalar dos puntos al respecto: I) si bien no se muestran los datos completos sobre los resultados de las otras variables involucradas en la medición de la pobreza, existe una relación entre las entidades federativas que tienen los peores resultados y un menor acceso a la educación como es el caso de Chiapas, Oaxaca y Guerrero. Por tal motivo, se puede deducir de manera preliminar la interdependencia simultánea de los derechos sociales; y II) en el caso de los 
tomadores de decisiones que elaboran políticas públicas educativas, si uno sigue una lógica lineal con estos datos y no toma en cuenta la complejidad del fenómeno educativo, se puede caer en el error de pensar que bastaría aumentar la cobertura en el nivel obligatorio para mejorar el derecho social a la educación.

\section{Método}

En la elaboración de este artículo surgió la siguiente duda: ¿cómo es posible aproximarnos al análisis y estudio del fenómeno educativo desde las ciencias de la complejidad? Para responderla, se tomó la decisión de utilizar una adaptación de la propuesta elaborada por Amozurrutia (2012), retroalimentado por Morin (1999). La Figura 2 parte del supuesto de que no hay realidad independiente de la observación, por lo que es necesario una epistemología que considere la función del observador y no de lo que es observado. Por tal motivo, el punto de partida es la observación como una condición necesaria pero no suficiente para analizar los fenómenos complejos. "Debemos aprender que la búsqueda de la verdad necesita la búsqueda y elaboración de meta-puntos de vista que permitan la reflexividad, que conlleven especialmente la integración del observador-conceptualizador en la observación-concepción y la ecologización de la observación-concepción en el contexto mental y cultural que es el suyo" (Morin, 2008, p.18).

En este sentido, Morin (1999) y Amozurrutia (2012) coinciden en la integración entre observador-concepción; así, el segundo propone observar desde el enfoque multidisciplinario y complejo.

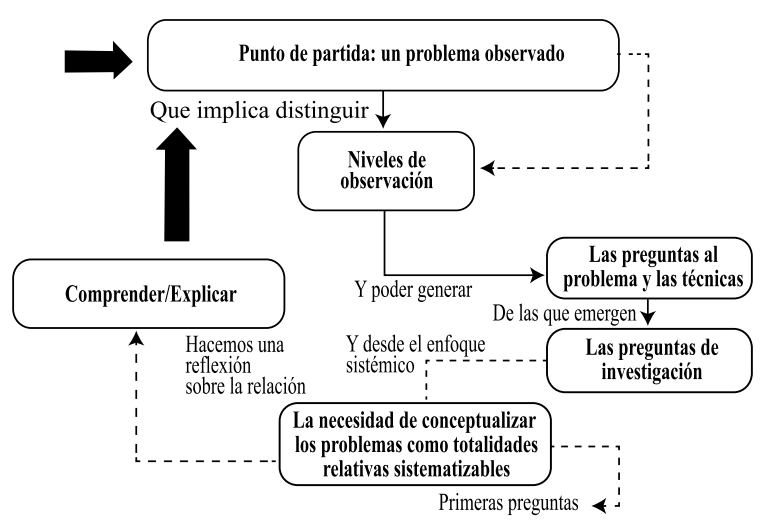

Figura 2. Propuesta para el estudio de fenómenos sociales desde las ciencias de la complejidad Fuente: Elaboración propia con base en Amozurrutia (2012)

Las observaciones sobre un fenómeno complejo permiten delimitar al menos una gran pregunta, conformada por una serie de preguntas desde diferentes disciplinas. Las respuestas conjugadas a ella se organizarán dentro de una estrategia conjunta que conducirá a una solución posible del problema. De este modo, la red de preguntas permite configurar un primer esquema de los temas y conceptos asociados a sus posibles soluciones; y es a partir de esta red que se establece un componente esencial para la estrategia de análisis del problema. Como puede verse, la epistemología es una posibilidad para vincular las ciencias de la complejidad con fenómenos sociales complejos como la pobreza, pues no hay realidad que sea independiente de la observación. Para Luhmann (2007), la observación es una operación autopoiética que consiste en el manejo y la manipulación de un sistema de diferencias que no constituyen un estado en sí, sino una operación que lleva a cabo un sistema. 
La observación es una acción del observador, una operación de tipo empírico que, a su vez, puede ser observada con una carga ontológica; Con base en Luhmann (2007), López (2008) propuso varios niveles de observación:

- Observaciones de primer orden. El observador mismo y su observación se mantienen inobservados porque no es necesario que el primero se distinga a sí mismo de aquello que observa; así se reduce la complejidad.

- Observaciones de segundo orden. La autoobservación supone el retorno de la observación sobre el sujeto que la ejercita, es decir, el observador necesita observarse para poder ver y observar la realidad.

- Observaciones de tercer orden. La observación de otras observaciones supone un nivel de autorreferencia distinto a la autoobservación, pues toma en cuenta otros observadores cuyas observaciones se desea analizar; supone el reconocimiento de la existencia de pluralidad de observaciones y sujetos que observan. En las observaciones de tercer orden se pueden detectar cegueras paradigmáticas, en las que se analiza la realidad solo desde el punto de vista de la especialidad.

- Observaciones de cuarto orden. Consisten en crear un nuevo paradigma que se adapte al contexto cultural; en este sentido, permiten analizar a complejidad.

- Observaciones de quinto orden. Realizan una comparación respetuosa con otras civilizaciones.

Con base en lo anterior, la pregunta que sirvió como detonante en esta investigación fue: ¿cómo se puede definir el derecho social a la educación? Tal pregunta permite realizar observaciones de primer orden sobre algunas propuestas disciplinares, en específico nos centramos en dos: I) la parte normativa que ofrece el derecho, que muchas veces condiciona las acciones del gobierno para resolver problemas como la pobreza. En este caso se tomaron como referencia la CPEUM y la LGDS, entre otras; II) la propuesta del CONEVAL, que, desde un enfoque estadístico, realiza una aproximación cuantitativa sobre qué tanto cumple el gobierno mexicano con sus objetivos educativos.

La metodología también propone la necesidad de conceptualizar el fenómeno educativo como totalidades relativas sistematizables, de modo que el concepto de totalidad se refiere a un conjunto de cualidades y características heterogéneas e interdependientes, organizadas en la concepción de sistema. Son relativas porque no pretenden tener todos los atributos posibles, sino los que le son más pertinentes para entender el fenómeno complejo:

El conocimiento pertinente debe enfrentar la complejidad. Complexus significa lo que está tejido junto; en efecto, hay complejidad cuando son inseparables los elementos diferentes que constituyen un todo (como el económico, el político, el sociológico, el sicológico, el afectivo, el mitológico) y que existe un tejido interdependiente, interactivo e interretroactivo entre el objeto de conocimiento y su contexto, las partes y el todo, el 
todo y las partes, las partes entre ellas. Por esto, la complejidad es la unión entre la unidad y la multiplicidad. (Morin, 1999, p.17)

La totalidad relativa no integra todos los atributos posibles de su dominio, sino la parte que ha sido observada y explicada por el investigador; incluye también las posibles relaciones de su interacción, no solo en su interior sino de aquellas derivadas de su acoplamiento con otros sistemas, dominios y totalidades con quienes convive. En este caso, la conceptualización del problema se puede realizar con un enfoque sistémico porque se asocia a la relación de acciones, comunicaciones, hechos y procesos dentro del complejo empírico, donde este complejo está configurado por la delimitación del conjunto de datos empíricos, derivados del registro de los observables; es importante señalar que definir los observables implica un conocimiento previo para establecer las relaciones básicas entre ellos, así como asociarlos a los hechos -ya como observables interpretados- y a los procesos, entendidos como una serie de cambios consecuentes de las relaciones causales entre los hechos. La totalidad relativa es comprensible: “(...) -como un sistema adaptativo- que en primera instancia se adapta a las necesidades de comprensión/explicación de los problemas en estudio y en segunda, contribuye en su transformación" (Amozurrutia, 2012, p.40).

\section{Resultados}

En la primera sección se presentó la propuesta del CONEVAL para medir a través de la variable de rezago educativo si existe o no carencia en el derecho social a la educación, pero, ¿realmente es la mejor aproximación para el análisis del derecho a la educación? El reto inicia cuando se intenta cuantificar un fenómeno multidimensional porque un indicador reduce la complejidad al no poder captar las relaciones con otras variables o con los efectos indirectos; por ejemplo, el acceso a los niveles de educación obligatorios puede omitir la calidad de la educación, las habilidades individuales, las redes sociales, la suerte, entre otros aspectos que también son importantes.

El uso de rezago educativo como indicador parece estar limitado por sí mismo para explicar el derecho social a la educación, por lo que es importante enriquecer el análisis con las propuestas de otras disciplinas como el derecho. El primer párrafo del artículo 26 de la Declaración Universal de los Derechos Humanos establece, en este sentido, que toda persona tiene derecho a la educación y que esta debe ser gratuita, al menos en la instrucción elemental y fundamental; de manera adicional, comprende también los siguientes estándares (Oficina del Alto Comisionado para los Derechos Humanos, 2004):

- Disponibilidad: los gobiernos tienen la responsabilidad de construir suficientes escuelas para garantizar que todos puedan asistir a una. Las escuelas deben ser equipadas con todos los materiales e instalaciones que necesitan para funcionar adecuadamente, como materiales de enseñanza, personal y 
maestros calificados, entre otros.

- Accesibilidad: las instituciones educativas deben ser accesibles para todos. En este sentido, necesitan contar con las condiciones mínimas de seguridad y ubicarse a distancia razonable de las comunidades; en este aspecto también se integra la igualdad de oportunidades educativas, donde la educación debe estar disponible para todos sin importar el nivel de ingresos.

- Aceptabilidad: los planes de estudio y los métodos pedagógicos de las escuelas deben ser aceptables para los padres de los niños y deben cumplir con las normas nacionales establecidas por el gobierno.

- Adaptabilidad: la educación debe ser adaptable a los cambios sociales e incluir los diferentes intereses sociales y culturales de los alumnos.

La educación tiene la importante función social de formar ciudadanos y ciudadanas capaces de ejercer sus derechos y obligaciones, ingresar al mercado laboral o desarrollar las habilidades mínimas que les permitan desenvolverse adecuadamente a lo largo de su vida. Por tal motivo, el derecho a la educación difícilmente se puede ejercer sin por lo menos tomar en cuenta, en el corto y mediano plazo, un análisis detallado de la calidad educativa, la pertinencia de los programas y contenidos curriculares, la deserción estudiantil y la cobertura (Hernández, 2009).

Tener acceso a la educación es una condición necesaria pero no suficiente para ejercer el derecho a la educación, porque también se debe tomar en cuenta su calidad, que depende de la eficiencia y eficacia del sistema educativo para generar un aprendizaje significativo que permita la participación de todos los individuos en la vida social, productiva y política (Hernández, 2009).

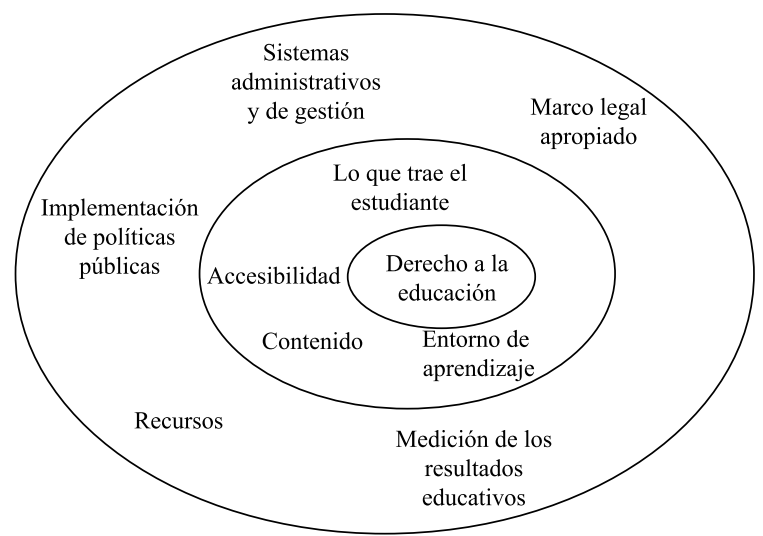

Figura 3. Enfoque sistémico para analizar el derecho a la educación con base en la calidad Fuente: Joy (2006).

En la Figura 3 se presenta una propuesta con un enfoque sistémico elaborada por Joy (2006), que pretende enriquecer la comprensión del derecho a la educación con énfasis en la calidad educativa. El primer nivel del sistema se centra en el estudiante, en quien se interrelacionan cinco elementos: I) la accesibilidad en un contexto de igualdad de oportunidades educativas; II) lo que atrae al estudiante como un participante activo y central en la construcción de su propio conocimiento; III) el contenido actualizado de lo que se ve en la currícula educativa; IV) los procesos de enseñanza-aprendizaje que necesitan una participación activa de los docentes, estudiantes y padres de familia, y V) el entorno de su aprendizaje que se relaciona con el entorno físico y psicosocial en el que se desenvuelve. 
El segundo nivel está relacionado con las competencias del Estado mexicano en materia educativa: I) estructuras y procesos administrativos y de gestión. En México la SEP es la encargada de regular, mientras que el sindicato y las escuelas son responsables de la implementación; II) implementación de políticas públicas donde el magisterio juega un papel importante; III) marco legal apropiado; IV) los recursos físicos, financieros y humanos suficientes para la labor educativa; y por último V), es importante contar con esquemas de medición de los resultados educativos, dado que la evaluación es importante no solo como herramienta de control, sino también es un espacio de participación que permite disminuir errores, establecer nuevas estrategias y hacer los procesos transparentes.

Como se evidencia, el acceso a la educación no puede asegurar por sí mismo el ejercicio correcto del derecho a la educación porque existen otros elementos y dimensiones importantes que están interrelacionados, como la calidad, los procesos, instituciones, recursos, entre otros. En este apartado solo se mencionaron de manera general algunas propuestas para la elaboración de otro tipo de indicadores, que tienen la ventaja de contar con fuentes de información de corte estadístico y permiten elaborar así un análisis que si bien no supera el problema de cualquier medición, ofrecen una visión complementaria del derecho a la educación.

Por ejemplo, para aproximarse a la disponibilidad y accesibilidad, la Secretaría de Educación Pública (SEP) y el Instituto Nacional de Estadística y Geografía (INEGI) elaboraron el Censo de Escuelas, Maestros y Alumnos de la Educación Básica y Especial; mientras que para conocer los avances en aceptabilidad y adaptabilidad se pueden utilizar herramientas como ENLACE, el Programa Internacional de Evaluación de los Alumnos (PISA), o cualquier otro instrumento que elabore el INEE para evaluar determinados resultados educativos en los estudiantes.

\section{Discusiones y conclusiones}

La pregunta central que este trabajo trató de abordar fue: ¿cómo se puede definir el derecho social a la educación? Para ello se adaptó una propuesta metodológica con base en Amozurrutia (2012), que analiza el derecho social a la educación. Con ella se plantea el hecho de que un observador es parte de lo que ve, u ocurre lo que Morin (1999) denomina reintroducción del cognoscente en todo conocimiento; esto último generó la necesidad de hacer observaciones de primero y segundo orden.

Los resultados del análisis del derecho a la educación en México indican: primero, que el carácter de indivisibilidad e interdependencia del derecho a la educación determina la complejidad, pues los derechos simplemente necesitan estas características o no son. De tal modo que su observación y su comprensión están enmarcadas por lo complejo; segundo: el paradigma no puede cambiar si el observador no se observa en la realidad donde está inmiscuido, esto es, que analizar un indicador de manera tradicional limita el espectro de visión al no considerar sus relaciones con otras variables. 
En términos generales, el análisis de la pobreza ha quedado supeditado al reduccionismo de las técnicas que propone el CONEVAL para la medición multidimensional de la pobreza, pero se necesita un enfoque más integral y complementario, un enfoque que posibilite la idea de que los derechos constitutivos del bienestar social se interrelacionan y son interdependientes, pues, como sabemos, para que haya autonomía en los derechos, estos deben ser dependientes del sistema que los contenga.

Las políticas públicas deben orientarse hacia un enfoque hologramático, donde no solo importe una de las partes (reduccionismo) ni tampoco el Todo (holismo), sino la reciprocidad existente entre ambos en un mismo proceso interrelacional. En el análisis del derecho a la educación, se pudo verificar, por el contrario, su "aislamiento" dentro del proceso gubernamental, en tanto este último combate claramente a la pobreza, pero ese derecho no puede generarse con las estrategias actuales, sino que necesita un campo de reflexión que incluya lo no lineal, lo no secuencial, en suma, políticas públicas reconocidas como unitas multiplex.

\section{Referencias}

Amozurrutia, J. (2012). Complejidad y sistemas sociales. Un modelo adaptativo para la investigación interdisciplinaria. Ciudad de México: Universidad Nacional Autónoma de México.

Consejo Nacional de Evaluación de la Política de Desarrollo Social (2014). Metodología para la medición multidimensional de la pobreza en México (segunda ed.). Ciudad de México: CONEVAL.

Hernández, G. (2009). Un nuevo rol docente en la era de las nuevas tecnologías. Cuadernos de Educación y Desarrollo, 1, 45-63.

Ibagón, N. J. (2015). La educación, un derecho que cuesta: dimensión fiscal y su relación con la política educativa en América Latina. Revista Educación y Humanismo, 17, 29-37.

Joy, M. (2006). ¿Qué es la calidad de la educación? (Desde la perspectiva de la UNESCO). En K. N. Ross, \& I. Jürgens, Estudios internacionales sobre la calidad de la educación. La planificación de su diseño y la gestión de su impacto (pp.41-53). París: UNESCO.

López, S. (2008). Reflexiones para la formación del psicólogo. Ciudad de México: Universidad Nacional Autónoma de México, FES-Iztacala.

Luhmann, N. (2007). Sistemas sociales: lineamientos para una teoría general. Barcelona: Anthropos Editorial.

Morin, E. (1999). Los siete saberes necesarios para la educación del futuro. París: Santillana UNESCO.

Morin, E. (2008). Introducción al pensamiento complejo. Barcelona: Gedisa.

Oficina del Alto Comisionado para los Derechos Humanos (2004). Los derechos humanos y la reducción de la pobreza: Un marco conceptual. Nueva York y Ginebra: Naciones Unidas. 